\title{
Clinical and radiological results with a 36-mm cobalt-chrome prosthetic head, cross-linked Durasul liners associated with Allofit cups: a more than I0-year follow-up period
}

HIP International

2020, Vol. 30(4) 446-45

(C) The Author(s) 2019

Article reuse guidelines:

sagepub.com/journals-permissions

DOl: 10.1177//1/20700019869829

journals.sagepub.com/home/hpi

(S)AGE

\author{
Pierre Georis, Thierry Thirion and Philippe Gillet
}

\begin{abstract}
Background: Highly cross-linked polyethylene is currently a common articulation surface used for total hip arthroplasty (THA).

Aim: The aim of the present study is in vivo assessment of highly cross-linked Durasul polyethylene linear and volumetric wear when associated with a 36-mm prosthetic femoral head.

Methods: We retrospectively reviewed clinical and radiographic data of 78 patients (8I hips) having primary THAs using Durasul liner combined with a 36-mm CoCr prosthetic head. All of them were followed for more than 10 years. Patient outcome was assessed with the Harris Hip Score (HHS) preoperatively and at last follow-up. 2-D prosthetic head penetration into polyethylene, 3D wear rates and cup migration were evaluated.

Results: The preoperative and last follow-up HHS were $50.43+/-10.42$ and $97.44+/-5.5$ I respectively. The annual penetration of the prosthetic head into Durasul ${ }^{\circledR}$ liner was $0.029+/-0.003 \mathrm{~mm}$. The annual linear penetration and volumetric wear extrapolation rates using Charnley and Ilchmann formulas were $37.84 \%$ and $57.76 \%$ respectively of that seen with conventional polyethylene liner. At last follow-up, the total loss of material in Durasul represents only $0.15 \%$ of the initial polyethylene mass. We did not observe any significant cup migration in the study group.

Conclusions: Results are promising, and we believe that these data authorise the continued use of highly cross-linked polyethylene liner associated with a 36-mm prosthetic head for total hip arthroplasties in older patients. More long-term follow-up studies are mandatory before we feel comfortable with the project of using cross-linked polyethylene in young and active patients instead of ceramic-on-ceramic bearings.
\end{abstract}

\section{Keywords}

Hip replacement, PE wear rate, volumetric wear

Date received: 25 October 2018; accepted: 3 February 2019

\section{Introduction}

For decades total hip arthroplasty (THA) has been 1 of the most successful surgical interventions in medicine. However the optimal bearing surface remains controversial. In 1995, Harris demonstrated that production of wear particulate debris is the main factor leading to periprosthetic osteolysis, aseptic loosening and long-term failure of an implant. ${ }^{1}$ The longevity of a THA with a metal-onpolyethylene bearing may be limited by wear of the polyethylene. $^{2-4}$ To reduce the volume of wear debris generated at the bearing surface and thereby improve the longevity of the prosthesis, several changes in the manufacturing process of conventional polyethylene have been made over the last 2 decades. Highly cross-linked polyethylene is currently a common articulation surface used for THA.

Orthopaedic Department, University Hospital Sart-Tilman, Liège, Belgium

\section{Corresponding author:}

Pierre Georis, CHU de Liège - Hôpital du Sart Tilman, University Hospital Orthopaedic Department, Avenue de l'Hòpital, I3, Liège 4000, Belgium.

Email: pierre.georis@chu.ulg.ac.be 
Durasul was developed in conjunction with W. Harris and the Massachusetts Institute of Technology. It has demonstrated improved wear performance over conventional polyethylene in laboratory testing. 5 The unique wearresistance of Durasul is achieved through an electronbeam radiation process ( $95 \mathrm{KGy}$ at $\sim 120^{\circ} \mathrm{C}$ ). Irradiation creates cross-links in the material's structure. The polyethylene is then subjected to a thermal treatment to remove free radicals (melting at $150^{\circ} \mathrm{C}$ for 2 hours). Sterilisation is obtained under ethylene oxide (EtO). ${ }^{6-8}$

On the other side dislocation represents, after aseptic loosening, the second most frequent complication in THA with occurrence of $2-3 \%$ following a primary THA and $10 \%$ in revision arthroplasties. ${ }^{9,10}$ Factors contributing to impingement and dislocation include soft tissue, bone components, orientation of the prosthesis and design of the implants. ${ }^{11}$ The range of motion (ROM) of the articulation is function of the effective position of the implants but also of the technical ROM influenced by the manufacturer's implant design. In order to obtain a sufficient postoperative hip mobility and stability; THA should provide a head-neck ratio of at least 2:1. ${ }^{12}$ The use of a large prosthetic head size could increase hip stability and greatly prevent the possible risk of dislocation. ${ }^{13,14}$

Highly cross-linked polyethylene theoretically allows the use of thinner inserts than with conventional polyethylene. Thinner inserts allow for larger diameter heads. This provides greater freedom of movement to patients and participates in reducing the risk of dislocation.

In 2001, Muratoglu et al. ${ }^{7}$ showed that cross-linked polyethylene volumetric wear is independent of the head diameter between 22 and $46 \mathrm{~mm}$. The insert must have a minimum 3-mm thickness. A minimum 5-mm thickness is suggested (7-mm for 22-mm head). ${ }^{7}$ This allows the use of a 36-mm prosthetic head combined with a minimum 52-mm diameter cup.

A possible complication related to implant thinness is liner fracture. Because the hip kinematics includes a piston effect up to $10 \mathrm{~mm}$, fracture toughness reduction of crosslinked polyethylene reaches more than $30 \%{ }^{15}$ In 2004 , Bradford et al. ${ }^{16}$ demonstrated that there was consistent evidence of surface cracking, abrasion, pitting and scratching on polyethylene inner surface on liners removed from early failure of defective metal shells after 10 months (recalled patients). The phenomenon was not predicted by in vitro hip simulator studies. The discrepancy between in vitro and in vivo wear surfaces may be due to variability in terms of in vivo lubrication and cyclic loading or may represent early surface damage mechanisms that are not well demonstrated by long term simulator studies.

The aim of the present study is in vivo assessment of highly cross-linked Durasul polyethylene linear and volumetric wear when associated with a $36-\mathrm{mm}$ prosthetic femoral head. We evaluated the behavior status of Durasul when it was combined with a $36-\mathrm{mm} \mathrm{CoCr}$ head or with a 28-mm CoCr prosthetic femoral head. We also hypothesise that Durasul combined with a $36-\mathrm{mm}$ head could produce less wear than a conventional polyethylene liner in association with a 28-mm ceramic femoral head.

\section{Material and methods}

Between May 2003 and September 200591 prostheses were implanted in 88 patients. All THA were performed by the same surgeon in the same hospital. Sex ratio male/ female was 30:58 with a mean age of 74.31 (range 62-89) years at the operation. To date, mean age of patients reaches 85.20years. Among them 20 patients died for unrelated cause to hip surgery but 10 of them after more than 10 years follow-up. These 10 patients were included to the study. We retrospectively reviewed clinical and radiographic data of 78 patients ( 81 hips) having primary THAs using Durasul liner (Zimmer Inc, Warsaw, IN, USA) combined with a 36- $\mathrm{mm}$ CoCr prosthetic head (Protasul). All of them were followed for more than 10 years. All patients received an Allofit acetabular cup (Zimmer Inc, Warsaw, IN, USA) of $52 \mathrm{~mm}(n=22), 54 \mathrm{~mm}(n=18)$, $56 \mathrm{~mm}(n=17), 58 \mathrm{~mm}(n=16), 60 \mathrm{~mm}(n=4), 62 \mathrm{~mm}$ $(n=3)$ and $64 \mathrm{~mm}(n=1)$. When osseous bone stock was sufficient, patients received a cementless Spotorno (CLS) stem $(n=17)$; the large majority of patients received a MS-30 cemented stem $(n=64)$. These 81 THA constituted the study group.

The first control group (control A) was composed of 16 THA with a Durasul liner combined with a 28-mm Protasul prosthetic head. The second control group (control B) was composed of 40 THA with a conventional polyethylene (UHMWPE) Sulene liner combined with a 28-mm Biolox prosthetic head. All patients from control groups were followed for more than 10 years (control A: 11.2 years, control B: 11.5 years).

Patient outcome was assessed with the Harris Hip Score (HHS) preoperatively and at last follow-up. We also collected adverse events and complications. ${ }^{17}$

Digitised radiographs taken at 6 weeks, 6 months, 1 year, 2 years, 5 years and at last follow-up were used for radiographic analysis. All measurements were performed on pelvic standing radiographs. In vivo penetration of the prosthetic head into the polyethylene was determined as $2 \mathrm{D}$ linear femoral head penetration on anteroposterior pelvis radiographs. The first 6 months after implantation, we took into account bedding-in penetration (difference between 6-week and 6-month radiographs). ${ }^{18}$ After this 6-month period, we interpreted measurement differences as true wear.

2D prosthetic head penetration into polyethylene (bedding-in and wear) assessment was performed using a specific analysis model created in the Imagika software (GreyStone, Paris, France). It uses an automatic edge detection to fit the contour of the prosthetic head and the cup. 
The contour of the prosthetic head was determined by using 3 points. The known diameter of the head $(36 \mathrm{~mm}$ or $28 \mathrm{~mm}$ ) is used for calibration. The diameter of the cup was determined by using 5 points. The software determines both centers (head and cup) and measures the distance between them on all consecutive films. 3-dimensional wear rates were calculated according Charnley $\left(V=\pi r^{2} w\right)$ and $\operatorname{Ilchmann}\left(V=\frac{r^{2} w}{2}\left(\pi+\pi \sin \beta+\frac{w}{r} \sin 2 \beta\right)+2 r D w \cos \beta\right)$ formulas. ${ }^{19,20}$ The Ilchmann formula considers the directional vector of linear penetration of the prosthetic head into the polyethylene (Beta angle). ${ }^{19}$

Cup migration was evaluated using the EBRA-CUP software. ${ }^{21}$

All groups (study group, control A and control B) were compared with unpaired Student t-test with a level of significance set at $5 \%$.

\section{Results}

In the study group, the preoperative and last follow-up HHS were $50.43+/-10.42$ and $97.44+/-5.51$ respectively. The same observation was made in both control groups. As in the large majority of hip procedures, patient's satisfaction is relatively high with a considerable improvement of life quality level. There was no significant difference (NSD) in HHS between all groups (Table 1).

Table I. Preoperative and last follow-up Harris Hip Scores in study and different control groups.

\begin{tabular}{lll}
\hline & Preoperative HHS & Last follow-up HHS \\
\hline Study group & $50.43+/-10.42$ & $97.44+/-5.5 I$ \\
Control A & $51.27+/-12.34$ & $97.23+/-4.94$ \\
Control B & $50.35+/-14.21$ & $96.47+/-6.34$ \\
\hline
\end{tabular}

HHS, Harris Hip Score.
Regarding bedding-in penetration of the prosthetic head into the polyethylene, we did not observe any significant difference between 3 groups (Table 2).

The annual penetration of the prosthetic head into Durasul liner was $0.029+/-0.003 \mathrm{~mm}$ and $0.032+/-$ $0.014 \mathrm{~mm}$ for the study group and the control A respectively with a $p$-value of 0.087 (NSD). Contrariwise, the annual penetration of the head in control $\mathrm{B}$ reached $0.083 \mathrm{~mm}$ with a $p$-value of 0.00027 (SD).

Mean Beta angle calculated was $62.0^{\circ}=/-6.4^{\circ}$ in study group, $64.0^{\circ}+/-7.2^{\circ}$ and $63.5^{\circ}+/-9.8^{\circ}$ in control $\mathrm{A}$ and control B respectively (NSD).

With the highly cross-linked Durasul polyethylene used in the study group, a 36-mm prosthetic head had unfavourable influence on the volumetric wear assessment compared with the use of a 28-mm prosthetic head (control A) but the wear volumes produced were relatively low (Table 3 ). The qualitative wear pattern of the highly cross-linked Durasul ${ }^{\circledR}$ polyethylene liner associated with a metallic $(\mathrm{CoCr}) 36-\mathrm{mm}$ Protasul prosthetic head was the same as that of the conventional polyethylene liner associated with a 28-mm Biolox prosthetic head. The annual linear penetration and volumetric wear extrapolation rates using Charnley and Ilchmann formulas were $37.84 \%$ and $57.76 \%$ respectively of that seen with conventional polyethylene liner (Table 4). At last follow-up, the total loss of material in Durasul represents only $0.15 \%$ of the initial polyethylene mass.

We did not observe any significant cup migration in the study group as well as in control groups. The absence of cup migration at last follow-up may indicate very low polyethylene wear rates (Table 5).

At last follow-up, no complication was observed related to implant thinness in the study group.

Unfortunately, we observed 1 traumatic dislocation in 1 patient. It was a 62-year-old female who received a MS-30 cemented stem combined with a $36-\mathrm{mm}$ prosthetic head associated with an Allofit 52-mm shell. This disoriented

Table 2. Bedding-in penetration of the prosthetic head into the polyethylene in all groups.

\begin{tabular}{lll}
\hline Study group & Control A & Control B \\
\hline $0.054+/-0.009 \mathrm{~mm}$ & $0.056+/-0.008 \mathrm{~mm}$ & $0.057+1-0.010 \mathrm{~mm}$ \\
Study group versus control A & $p=0.36$ & NSD \\
Study group versus control B & $p=0.42$ & NSD \\
Control A versus control B & $p=0.61$ & NSD \\
\hline
\end{tabular}

NSD, no significant difference.

Table 3. Volumetric wear extrapolation rates using Charnley and Ilchmann formulas in all groups.

\begin{tabular}{lll}
\hline & $\begin{array}{l}\text { Annual volumetric wear } \\
\text { extrapolation (Charnley) }\end{array}$ & $\begin{array}{l}\text { Annual volumetric wear } \\
\text { extrapolation (Ilchmann) }\end{array}$ \\
\hline Study group & $29.52+1-6.01 \mathrm{~mm}^{3}$ & $27.81+1-4.12 \mathrm{~mm}^{3}$ \\
Control A & $19.70+/-17.28 \mathrm{~mm}^{3}$ & $13.46+/-6.54 \mathrm{~mm}^{3}$ \\
Control B & $51.11+/-68.75 \mathrm{~mm}^{3}$ & $48.55+/-84.47 \mathrm{~mm}^{3}$ \\
\hline
\end{tabular}


Table 4. Statistical analysis of volumetric wear rate differences between all groups.

\begin{tabular}{lll}
\hline & Charnley formula & Ilchmann formula \\
\hline Study group versus control A & $p=0.0046(S D)$ & $p=0.0004($ SD) \\
Study group versus control B & $p=0.000042$ (SD) & $p=0.00075$ (SD) \\
Control A versus control B & $p=0.000002$ (SD) & $p=0.0000064$ (SD) \\
\hline
\end{tabular}

$\mathrm{SD}$, significant difference.

Table 5. Medial and cranial cup migrations in all groups.

\begin{tabular}{lll}
\hline & Medial cup migration & Cranial cup migration \\
\hline Study group & $0.09+/-0.01 \mathrm{~mm}$ & $0.13+/-0.02 \mathrm{~mm}$ \\
Control A & $0.10+/-0.05 \mathrm{~mm}$ & $0.12+/-0.02 \mathrm{~mm}$ \\
Control B & $0.11+/-0.07 \mathrm{~mm}$ & $0.14+/-0.03 \mathrm{~mm}$ \\
\hline
\end{tabular}

patient had fallen from a chair at 6 months after primary intervention. She was reduced under general anaesthesia in the emergency room and she is doing well since without any sign of polyethylene wear or cup migration.

Recently, we observed a stem subsidence with probable loosening in an 85-year-old female. She received 11.5 years before a MS-30 cemented stem with a $36-\mathrm{mm}$ prosthetic head associated with a 54-mm Allofit cup. However, there is no sign of polyethylene wear or cup migration. Osseous scintigraphy confirms the loosening of the femoral implant. This patient is under further investigation for septic stem loosening due to probable infection.

\section{Discussion}

The main purpose of the present study was to assess and quantify wear rates of Durasul insert combined with a 36-mm Protasul prosthetic head. We also attempt to demonstrate that the use of larger prosthetic head did not influence the linear prosthetic head penetration into the highly cross-linked Durasul polyethylene compared with the use of smaller prosthetic head combined with conventional polyethylene. In parallel, we tried to verify if the use of larger than 28-mm prosthetic head had a favourable effect on dislocation rate.

Regarding bedding-in penetration of the prosthetic head into the polyethylene, we did not observe any difference whatever the prosthetic head diameter or the polyethylene type used. This is in opposition to the observations made by Olyslaegers et al. ${ }^{22}$ for whom bedding-in penetration occurs in the first 2 years following implantation. They suggested that cross-linked polyethylene liners adapt more slowly to femoral head penetration. These observations seem to be confirmed by Digas et al. ${ }^{23}$ and Manning et al. ${ }^{24}$

Some studies showed that cross-linked polyethylene inserts have a higher resistance to long-term wear than conventional polyethylene inserts..$^{25,26}$ This is corroborated by the results published of mid-term follow-up studies. ${ }^{27-31}$
The present study confirms the wear rate reduction when using a cross-linked polyethylene insert compared with conventional polyethylene liner. We objectivised wear rates of $27.81 \mathrm{~mm}^{3}$ and $13.46 \mathrm{~mm}^{3}$ annually when using a Durasul liner combined with a $36-\mathrm{mm}$ or a $28-\mathrm{mm}$ Protasul prosthetic head instead of $48.55 \mathrm{~mm}^{3}$ annual wear rate when using conventional Sulene liner associated with a 28-mm Biolox prosthetic head. The use of Durasul liners lead to a drastic wear rate reduction of $37.84 \%$ and $57.76 \%$ respectively of that seen with the Sulene liner.

Other authors advocated that the use of a cross-linked polyethylene liner instead of a conventional type may reduce the wear rate up to $95 \%$ (range 70-95) $23,32-34$ The results of our study are les optimist but we used a $36-\mathrm{mm}$ prosthetic head while other authors reported observations based on the use of a $28-\mathrm{mm}$ or a $32-\mathrm{mm}$ prosthetic femoral head. It is clearly demonstrated that the larger prosthetic heads increases the friction surface in contact with the inner part of the polyethylene insert and at the same time produces more wear particles than smaller prosthetic heads. ${ }^{35}$ Despite the use of a large ball, our study demonstrates less linear head penetration into the Durasul polyethylene insert than other authors using the same socket. $^{23,26,30}$ All these articles support the steady-state behavior of the Durasul liner and the hope of promising results in the future.

More recently, 2 meta-analyses clearly demonstrated that cross-linked polyethylene decreases the prosthetic head penetration rate in the liner and decreases the volumetric wear rate but surprisingly the hypothesis that crosslinked polyethylene has an advantage over conventional polyethylene in terms of reducing osteolysis or revision rate is contradicted. ${ }^{36,37}$

This is a retrospective study of more than 10 years follow-up, and unfortunately, we were not able to produce an equal amount of THA in the 3 groups. However, Sulene polyethylene liners constituting control B group have been used for a long time and its wear rate is well known. The control A group was composed of Durasul liners combined with a $28-\mathrm{mm}$ prosthetic head. The small number of cases in this group is due to the preferential use of a larger prosthetic head in the large majority of the cases in prevision of limitation of the dislocation risk. A $28-\mathrm{mm}$ prosthetic head was only used with cups less than 52-mm. The main purpose of this study was to measure wear in the Durasul acetabular liner and to determine if there was 
really a difference between cross-linked and conventional polyethylene whatever the prosthetic head diameter used. Our results demonstrate a drastic reduction in linear penetration of the prosthetic head $(36 \mathrm{~mm}$ or $28 \mathrm{~mm})$ into the highly cross-linked Durasul polyethylene compared with the conventional Sulene polyethylene combined with a 28-mm Biolox prosthetic head.

The most important factors influencing postoperative mobility and stability of the prosthetic hip are the head diameter and the neck geometry (head-neck ratio of 2:1). ${ }^{12}$ Increasing the prosthetic head diameter enlarges the range of motion of the hip. In addition, stability is increased as a result of the larger prosthetic head being set deeper into the acetabular component compared to that of the smaller head size. As a result, the risk of impingement is lower and the risk of dislocation is reduced. Because of the relatively low wear rates objectivised in cross-linked polyethylene, the use of $36-\mathrm{mm}$ prosthetic head becomes possible. ${ }^{38} \mathrm{We}$ are in agreement with the results published by Geller et al. ${ }^{39}$ who demonstrated in 2006 that the use of large prosthetic heads $(>32 \mathrm{~mm})$ with a highly cross-linked polyethylene could be considered in patients with increased risk for dislocation. In their series of 45 hips, they mentioned 1 dislocation due to a grossly malpositioned acetabular component necessitating early revision. ${ }^{39}$ In our study group, a disoriented patient had fallen from a chair at 6 months after primary intervention. The prosthetic hip was reduced under general anesthesia in the emergency room without implant revision because we did not objectivise any sign of polyethylene wear or cup and stem migration.

In the whole series, no cup migration was observed. No osteolytic area or radiolucent lines in the 3 cup's Gruen zones has been put in evidence.

Fortunately, we did not observe any complications related to implant thinness like liner fracture as predicted by Pruitt in $2005 .{ }^{15}$ However, in our study group we must deplore a stem subsidence with probable septic loosening in 1 patient with no sign of polyethylene wear or cup migration.

Results are promising and we believe that these data authorise the continued use of highly cross-linked polyethylene liner associated with a $36-\mathrm{mm}$ prosthetic head for total hip arthroplasties in older patients. To date, in our institution, the use of Durasul implants is a daily activity. Compared to this study group, we decided to change the use of a metallic prosthetic head (Protasul $36 \mathrm{~mm}$ ) by a ceramic one (Biolox Delta $36 \mathrm{~mm}$ ). Dahl et al. ${ }^{40}$ clearly demonstrated that alumina heads performed better than cobalt-chrome heads after 10-year follow-up period. These findings support the use of alumina heads to reduce polyethylene wear in THA.

More long-term follow-up published studies are mandatory to check the validity of this hypothesis and before we feel comfortable with the project of using highly crosslinked polyethylene in young and active patients instead of ceramic-on-ceramic bearings. Considering that the type of implant used in our study group is mainly reserved for the elderly, obtaining long-term results with large cohort seems to be utopic.

\section{Declaration of conflicting interests}

The author(s) declared no potential conflicts of interest with respect to the research, authorship, and/or publication of this article.

\section{Funding}

The author(s) received no financial support for the research, authorship and/or publication of this article.

\section{References}

1. Harris WH. The problem is osteolysis. Clin Orthop Relat Res 1995; 311: 46-53.

2. Maloney WJ, Galante JO, Anderson M, et al. Fixation, polyethylene wear, and pelvic osteolysis in primary total hip replacement. Clin Orthop Relat Res 1999; 369: 157-164.

3. Sochart DH. Relationship of acetabular wear to osteolysis and loosening in total hip arthroplasty. Clin Orthop Relat Res 1999; 363: 135-150.

4. Oparaugo PC, Clarke IC, Malchau H, et al. Correlation of wear debris-induced osteolysis and revision with volumetric wear-rates of polyethylene: a survey of 8 reports in the literature. Acta Orthop Scand 2001; 72: 22-28.

5. Harris WH and Muratoglu OK. A review of current crosslinked polyethylene's used in total joint arthroplasty. Clin Orthop Relat Res 2005; 430: 46-52.

6. Muratoglu OK, Bragdon CR, O'Connor DO, et al. Unified wear model for highly crosslinked ultra-high molecular weight polyethylenes (UHMWPE). Biomaterials 1999; 20: 1463-1470.

7. Muratoglu OK, Bragdon CR, O'Connor DO, et al. A novel method of cross-linking ultra-high-molecular-weight polyethylene to improve wear, reduce oxidation, and retain mechanical properties. Recipient of the 1999 HAP Paul award. J Arthroplasty 2001; 16: 149-160.

8. McKellop H, Shen FW, Lu B, et al. Effect of sterilization method and other modifications on the wear resistance of acetabular cups made of ultra-high molecular weight polyethylene. A hip simulator study. J Bone Joint Surg Am 2000; 82: $1708-1725$.

9. Giurea A, Zehetgruber H, Funovics P, et al. Risk factors for dislocation of a cement-free total hip endoprosthesis a statistical analysis. Z Orthop Ihre Grenzgeb 2001; 139: 194-199.

10. Morrey BF. Difficult complications after total hip joint replacement: dislocation. Clin Orthop Relat Res 1997; 344: 179-187.

11. Siebenrock KA and Ganz R. The impingement problem in total hip arthroplasty. In: Reiker C (ed) World tribology forum in arthroplasty. Berne: Huber, 2001, pp.47-52.

12. Bader R, Scholz R, Steinhauser E, et al. The influence of head and neck geometry on stability of total hip replacement: a mechanical test study. Acta Orthop Scand 2004; 75: 415-421. 
13. Crowninshield RD, Laloney WJ, Wentz $\mathrm{DH}$, et al. Biomechanics of large femoral heads. What they do and don't do. Clin Orthop Relat Res 2004; 429: 102-107.

14. Berry DJ, von Knoch M, Schleck CD, et al. Effect of femoral head diameter and operative approach on risk of dislocation after total hip arthroplasty. J Bone Joint Surg Am 2005; 87: 2456-2463.

15. Pruitt LA. Deformation, yelding, fracture and fatigue behavior of conventional and highly cross-linked ultra high molecular weight polyethylene. Biomaterials 2005; 26: 905-915.

16. Bradford L, Baker DA, Graham J, et al. Wear and surface cracking in early retrieved cross-linked polyethylene acetabular liners. J Bone Joint Surg Am 2004; 86: 1271-1282.

17. Harris WH. Traumatic arthritis of the hip after dislocation and acetabular fractures: treatment by mold arthroplasty. An end-result study using a new method of results evaluation. $J$ Bone Joint Surg Am 1969; 51: 737-755.

18. Sychterz CJ, Engh CA Jr, Yang A, et al. Analysis of temporal wear patterns of porous-coated acetabular components: distinguishing between true wear and so-called bedding-in. J Bone Joint Surg Am 1999; 81: 821-830.

19. Ilchmann T, Reimold $M$ and Müller-Schauenburg W. Estimation of the wear volume after total hip replacement. A simple access to geometrical concepts. Med Eng Phys 2008; 30: 373-379.

20. Wu JS, Hsu SL and Chen JH. Evaluating the accuracy of wear formulae for acetabular cup liners. Med Biol Eng Comput 2010; 48: 157-165.

21. Ilchmann T, Kesteris U and Wingstrand H. EBRA improves the accuracy of radiographic analysis of acetabular cup migration. Acta Orthop Scand 199; 69: 119-124.

22. Olyslaegers C, Defoort K, Simon JP, et al. Wear in conventional and highly cross-linked polyethylene cups. $J$ Arthroplasty 2008; 23: 489-494.

23. Digas G, Kärrholm J, Thanner J, et al. 5-year experience of highly cross-linked polyethylene in cemented and uncemented sockets: two randomized studies using radiostereometric analysis. Acta Orthop 2007; 78: 746-754.

24. Manning DW, Chiang PP, Martell JM, et al. In vivo comparative wear study of traditional and highly cross-linked polyethylene in total hip arthroplasty. J Arthroplasty 2005; 20: 880-886.

25. Engh CA Jr, Hopper RH Jr, Huynh C, et al. A prospective, randomized study of cross-linked and non-cross-linked polyethylene for total hip arthroplasty at 10-year follow-up. $J$ Arthroplasty 2012; 27(Suppl. 8): 2-7.e1.

26. García-Rey E, García-Cimbrelo E and Cruz-Pardos A. New polyethylenes in total hip replacement: a ten- to 12-year follow-up study. J Bone Joint J 2013; 95-B: 326-332.

27. Bragdon CR, Kwon YM, Geller JA, et al. Minimum 6-year follow-up of highly cross-linked polyethylene in THA. Clin Orthop Relat Res 2007; 465: 122-127.
28. Glyn-Jones S, Isaac S, Hauptfleisch J, et al. Does highly cross-linked polyethylene wear less than conventional polyethylene in total hip arthroplasty? A double-blind, randomized, and controlled trial using roentgen stereophotogrammetric analysis. J Arthroplasty 2008; 23: 337-343.

29. Beksaç B, Salas A, González Della Valle A, et al. Wear is reduced in THA performed with highly cross-linked polyethylene. Clin Orthop Relat Res 2009; 467: 1765-1772.

30. Triclot P, Grosjean G, El Masri F, et al. A comparison of the penetration rate of two polyethylene acetabular liners of different levels of cross-linking: a prospective randomized trial. J Bone Joint Surg Br 2007; 89: 1439-1445.

31. Calvert GT, Devane PA, Fielden J, et al. A double-blind, prospective, randomized controlled trial comparing highly cross-linked and conventional polyethylene in primary total hip arthroplasty. J Arthroplasty 2009; 24: 505-510.

32. Heisel C, Silva M and Schmalzried TP. In vivo wear of bilateral total hip replacements: conventional versus crosslinked polyethylene. Arch Orthop Trauma Surg 2005; 125: 555-557.

33. McKellop H, Shen FW, DiMaio W, et al. Wear of gamma cross-linked polyethylene acetabular cups against roughened femoral balls. Clin Orthop Relat Res 1999; 369: 73-82.

34. Muratoglu OK, Greenbaum ES, Bragdon CR, et al. Surface analysis of early retrieved acetabular polyethylene liners: a comparison of conventional and highly cross-linked polyethylenes. J Arthroplasty 2004; 19: 68-77.

35. Burroughs BR, Rubash HE and Harris WH. Femoral head size larger than $32 \mathrm{~mm}$ against highly cross-linked polyethylene. Clin Orthop Relat Res 2002; 405: 150-157.

36. Kuzyk PR, Saccone M, Sprague S, et al. Cross-linked versus conventional polyethylene for total hip replacement: a meta-analysis of randomised controlled trials. $J$ Bone Joint Surg Br 2001; 93: 593-600.

37. Shen C, Tang ZH, Hu JZ, et al. Does cross-linked polyethylene decrease the revision rate of total hip arthroplasty compared with conventional polyethylene? A meta-analysis. Orthop Traumatol Surg Res 2014; 100: 745-750.

38. Muratoglu OK, Bragdon CR, O'Connor D, et al. Larger diameter femoral heads used in conjunction with a highly cross-linked ultra-high molecular weight polyethylene: a new concept. $J$ Arthroplasty 2001; 16(Suppl. 1): 24-30.

39. Geller JA, Malchau H, Bragdon C, et al. Large diameter femoral heads on highly cross-linked polyethylene: a minimum 3-year results. Clin Orthop Relat Res 2006; 447: 53-59.

40. Dahl J, Söderlund P, Nivbrant B, et al. Less wear with aluminium-oxide heads than cobalt-chrome heads with ultra high molecular weight cemented polyethylene cups: a tenyear follow-up with radiostereometry. Int Orthop 2012; 36: 485-490. 